\title{
COMPARISON OF PARTICLE SIZE DISTRIBUTION MODELS FOR POLYMER SWELLING
}

\author{
ÁdÁM WIRNHARDT *1 AND TAMÁS VARGA ${ }^{1}$ \\ ${ }^{1}$ Department of Process Engineering, University of Pannonia, Egyetem u. 10, Veszprém H-8200, \\ HUNGARY
}

In polymer technologies, various particle shapes and size distributions can be found. One of these are heterodisperse polymer beads. The capabilities of polymer swelling can be used in industries, e.g in the production of ion-exchange resins, to intensify specific technological steps such as sulphonation in the manufacturing process of ion-exchange resins. According to the literature different approaches can be used to create models for describing the behavior of disperse systems, of which the simplest models are the particle size distribution models for a given state of the solid phase. The aim of our examination was to compare and evaluate these simple models in terms of modeling polymer swelling. Hence, most of these models examine how each of the investigated models can be applied to approximately describe growth in a heterodisperse polymer system and how the identified model parameters in each time step could be interpreted. All the models were fitted to generate particle size distributions based on a swelling rate constant. The swelling of a styrene divinylbenzene-based copolymer was chosen as the basis of our examination. A model is proposed that is capable of describing the changes in the size of beads over time in this system.

Keywords: polymer swelling, particle size distribution, heterodisperse polymer beads, modeling, ion-exchange resin

\section{Introduction}

Polymer beads are used as raw materials in a wide range of technologies, e.g. in the production of ion-exchange resins. Before the chemical modification of polymer beads, they are often swollen with different types of swelling agents such as dichloroethane, dichloromethane, toluene, etc.

Monodisperse and heterodisperse types of beads are known in polymer technologies. The process of the swelling of monodisperse beads is easily measurable and easily predictable. The production of monodisperse polymers is a more expensive process than the production of heterodisperse beads, which makes heterodisperse polymer beads a more popular form.

Heterodisperse polymer beads exhibit a closely normal distribution in terms of particle size. The prediction of the swelling of these particle systems is more difficult because the different beads can swell at significantly different rates due to the change in the specific area of each bead.

The swelling of the polymer network system has already been a subject of interest. Painter and Shenoy[1] considered the chemical properties of polymers. Schott [2] described the kinetics of polymer swelling. First order and second order kinetics were founded by him. A

\footnotetext{
*Correspondence: wirnhardt.adam@fmt.uni-pannon.hu
}

swelling model was formulated by Sweijen et al. [3] according to the diffusion properties of components in the polymer matrix. These models are capable of describing the swelling of polymer networks, but because of its complexity they are hard to apply in any kind of optimization process. Hence, the simplest particle size distribution (PSD) model, which can describe the swelling of polymers over time using the least number of parameters, can be of interest from a process intensification point of view.

Bayat et al. collected PSD models from the last seventy years $[4,5]$. Thirty-five models were listed. These models describe the cumulative mass fraction of polymers as a function of the diameter of polymer beads. The models contain one, two, three or four unknown parameters, which can be identified with a specific polymer fraction. A hyperbolic tangent distribution [6,7] PSD model composed of four parameters was added to this list by us.

This study is the first step in the process of developing this model which focuses on the investigation of the swelling phenomena of the styrene divinylbenzene copolymer system. Therefore, our focus is on identifying a simple PSD model which is capable of describing the swelling of heterodisperse polymer beads. Hence, all the previously mentioned PSD models are investigated and compared. Our aim was not only to identify a PSD model which is capable of describing the distribution of the investigated polymer system but to find a PSD model which 
exhibits a correlation between changes made to parameters and particle size.

\section{Experimental}

For the modelling of heterodisperse polymer systems physical experiments with regard to swelling should be performed to obtain the data necessary to validate the model. In our case, with the lack of experiments, the data were generated from a model implemented and solved in MATLAB. A code was made in MATLAB for the generation of these distributions. In the developed model, the swelling of polymer beads with a given theoretical rate of growth was calculated. The volume of each bead increased at the same rate. Hence, the diameter of the beads does not influence the rate of growth and the tension caused by the swelling process of the polymer beads is neglected in this investigation. The following simplifications were implemented in the model:

1. The shape of the polymer beads is a perfect sphere.

2. The particles swell until they reach a steady state.

3. The swelling rate of particles is constant until a steady state is achieved.

4. The number of particles is constant.

5. The initial PSD of the beads is close to normal.

To generate the distributions after different durations of swelling an initial unswollen distribution is required. The initial distribution was calculated by MATLAB from a picture of heterodispersed particles. The Varion KS preform styrene divinylbenzene copolymer was used for the zero-time distribution. The polymer beads were identified by a circle detection algorithm and the size distribution of the detected particles was calculated using a reference particle.

From the initial state, the size of the polymer particles starts to increase by applying a swelling agent to the system. The size of the particles increases until a steady state is achieved. The steady state in this case means that the size of the particles grows until a state when the amount of infiltration of the swelling agent is equal to the outcome amount. The size of the particles can increase until a maximum is reached because of the internal tension of the polymer. The data are generated using the parameters of the steady state. These parameters are the swelling rate $(p[-])$ and time required $(t[\mathrm{sec}])$.

PSDs were generated at different times during the swelling process. The next step was to examine the PSD models to determine if they were able to describe the distribution in every instant.

\subsection{PSD models}

In this study only PSD models that are capable of describing cumulative mass fraction distribution were investigated. Altogether five models with one parameter, twelve with two, two with three and four with four were examined. They are collected in Table 1.

The cumulative mass fraction of particles is denoted by $P(d)$, the maxima and minima of the particle size range are represented by $d_{\max }$ and $d_{\min }$, respectively, and the particle diameter [mm] is denoted by $d$. The models were fitted to all the distributions collected over time using extreme value problem solver algorithms in MATLAB.

\subsection{Theoretical methodologies}

Two types of extreme value problem-solving methods were applied to fit the PSD models. One is a local extreme value problem solver known as the Nelder-Mead simplex algorithm and its function "fminsearch" to implement it in MATLAB. The other one is a global extreme value problem solver called "NOMAD" [8]. They are both components of the MATLAB toolkit.

MATLAB 2011b was applied in all modelling steps. The minimum difference was sought between the generated distribution data and calculated distributions based on each model. The parameters of PSD models were the results of this search. In every case, the goodness of fit was measured. For each model, every sample time was considered and the difference examined using the mean absolute difference. The average of the mean absolute difference of the percentage difference was calculated for every function. The extreme value problem solver attempted to find the minimum of the following equation

$$
E_{t}=\frac{\left|P(d)_{t}^{\prime}-P(d)_{t}\right|}{n_{d}}
$$

where the mean absolute difference between the generated and calculated distribution is $E_{t}$ at instant $t$. The calculated distribution is denoted by $P(d)_{t}^{\prime}$ and the generated distribution by $P(d)_{t}$ at instant $t$. The number of items of data is represented by $n_{d}$.

\subsection{Model selection}

A selection could be made according to the average percentage differences. The selection was carried out with a criterion. This criterion was an average percentage difference of five percent because under this value the difference is not considerable but over it an unacceptable fit is shown. Three different models, namely the Rosin-Rammler, the Exponential-power_Pasikatan and the Logarithm-Zhuang models fitted to the generated data are shown in Fig. 1. The goodness of fit for these three models is $1 \%, 5 \%$ and $11 \%$, respectively. As can be seen the differences of $1 \%$ and $5 \%$ are negligible, and are only noticeable at diameters in excess of $0.7 \mathrm{~mm}$. However, a considerable difference can be observed between the models with fits of $5 \%$ and $11 \%$. It can be seen that a goodness of fit of under $5 \%$ is appropriate. 
Table 1: The investigated PSD models

\begin{tabular}{|c|c|c|}
\hline & Name & Model \\
\hline \multicolumn{3}{|c|}{1 parameter $\left(k_{1}\right)$} \\
\hline 1 & Gaudy-Meloy & $P(d)=1-\left(1-d / d_{\max }\right)^{k_{1}}$ \\
\hline 2 & Nesbitt-Breytenbach & $\begin{array}{l}P(d)=10\left[\left(1 /\left(k_{1}+1\right)\right](d / 2)^{k_{1}+1}\right. \\
+\left[0.1-1 /\left(k_{1}+1\right)\right](d / 2)^{1 /\left[1 /\left(k_{1}+1\right)-0.1\right]}\end{array}$ \\
\hline 3 & Rosin-Rammler & $P(d)=1-\exp \left(-k_{1} d\right)$ \\
\hline 4 & Jaky & $P(d)=\exp \left\{-\left(1 / k_{1}^{2}\right)\left[\ln \left(d / d_{\max }\right)\right]^{2}\right\}$ \\
\hline 5 & Schumann & $P(d)=\left(d / d_{\max }\right)^{k_{1}}$ \\
\hline \multicolumn{3}{|c|}{2 parameters $\left(k_{1}, k_{2}\right)$} \\
\hline 6 & Power low- Paskikatan & $P(d)=\left[k_{1} /\left(1-k_{2}\right)\right] d^{1-k_{2}}$ \\
\hline 7 & van Genuchten & $P(d)=\left[1+\left(k_{1} / d\right)^{k_{2}}\right]^{1 / k_{2}-1}$ \\
\hline 8 & Rosin-Rammler & $P(d)=1-\exp \left(-k_{1} d^{k_{2}}\right)$ \\
\hline 9 & Fractal & $\begin{array}{l}P(d)=\exp \left\{\ln \left(k_{2}\right)\right)+ \\
\left.\left[\left(3 k_{1}^{2}-13 k_{1}+14\right) /\left(k_{1}^{2}-5 k_{1}+4\right)+1\right] \log (d)\right\}\end{array}$ \\
\hline 10 & Power low - Gimenez & $P(d)=k_{1} d^{k_{2}}$ \\
\hline 11 & BEST & $P(d)=\left[1+\left(k_{1} / d\right)^{k_{2}}\right]^{2 / k_{2}-1}$ \\
\hline 12 & Bennet & $P(d)=k_{1} k_{2} d^{k_{1}-1} \exp \left(-k_{2} d^{k_{1}}\right)$ \\
\hline 13 & Exponential power- Pasikatan & $P(d)=\exp \left(-k_{1} d^{k_{2}}\right)$ \\
\hline 14 & Logarithm-Zhuang & $P(d)=k_{1} \ln (d)+k_{2}$ \\
\hline 15 & Log-exp-Kolev & $P(d)=k_{1} \exp \left[k_{2} \log (d)\right]$ \\
\hline 16 & Weibull-2par & $P(d)=1-\exp \left[-\left(d / k_{1}\right)^{k_{2}}\right]$ \\
\hline 17 & Lognormal- Zobeek & $P(d)=1 /\left\{k_{1}(2 \pi)^{1 / 2} \exp \left[-\left(\log (d)-k_{2}\right)^{2} /\left(2 k_{1}^{2}\right)\right]\right\}$ \\
\hline \multicolumn{3}{|c|}{3 parameters $\left(k_{1}, k_{2}, k_{3}\right)$} \\
\hline 18 & S-Curve: Vipulanandan Ozgurel & $P(d)=100 \exp \left\{-k_{1}\left[k_{2} \ln (d / 0.001)^{d / k_{3}}\right]\right\}$ \\
\hline 19 & Weibull-3par & $P(d)=k_{1}-\exp \left[-\left(d / k_{2}\right)^{k_{3}}\right]$ \\
\hline \multicolumn{3}{|c|}{4 parameters $\left(k_{1}, k_{2}, k_{3}, k_{4}\right)$} \\
\hline 20 & Gompertz & $P(d)=k_{1}+k_{2} \exp \left\{-\exp \left[-k_{3}\left(d-k_{4}\right)\right]\right\}$ \\
\hline 21 & Weibull-4par & $\begin{array}{l}P(d)=k_{3}+\left(1-k_{3}\right)\left[1-\exp \left(-k_{1} k_{4}^{k_{2}}\right)\right] \\
\text { where } k_{4}=\left(d-d_{\min }\right) /\left(d_{\max }-d_{\min }\right)\end{array}$ \\
\hline 22 & Fredlund & $\begin{array}{l}P(d)=\left\{1 /\left[\ln \left(\exp (1)+\left(k_{1} / k_{2}\right)^{k_{2}}\right)\right]^{k_{3}}\right\} \\
\left\{1-\left[\ln \left(1+k_{4} / d\right) / \ln \left(1+k_{4} / 0.001\right)\right]^{7}\right\}\end{array}$ \\
\hline 23 & Tanh & $\begin{array}{l}\text { if } 0<k_{2}+k_{3} d \text { then } P(d)=\left\{\tanh \left[\left(k_{2}+k_{3} d\right)^{k_{4}}\right]\right\}^{k_{1}} \\
\text { otherwise } P(d)=0\end{array}$ \\
\hline
\end{tabular}




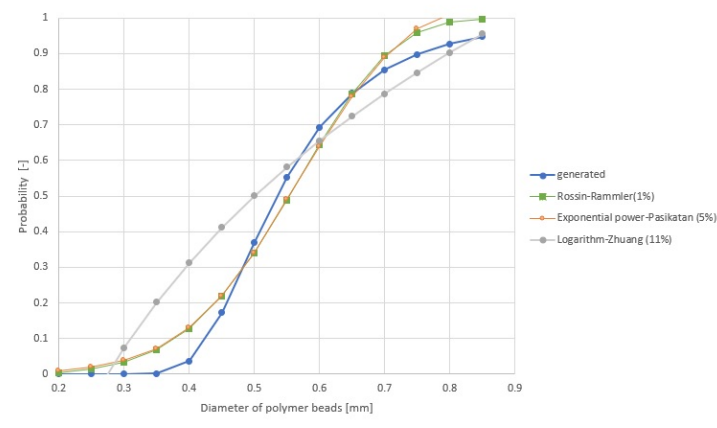

Figure 1: The cumulative distribution of the RosinRammler (1\%), Exponential-power Pasikatan (5\%) and Logarithm-Zhuang (11\%) models as well as the generated data.

\subsection{Parameter correlation}

The next step in the modelling process was the investigation into how the model parameters of each simple PSD model can be fitted into a monotonous series over time. Based on this experiment, these simple models can be applied to describe the swelling of specific material systems over time.

By depicting the parameters of the distribution functions as a function of time, a statement can be made for the models if they were capable of this task. Assuming that the correlation is linear for every model parameter, in each PSD model it can be calculated as follows:

$$
k_{x}=A_{x}+B_{x} t
$$

where the parameter of the PSD model is denoted by $k_{x}$, the parameters of the $k$ parameter are $A$ and $B$, and the time passed is $t$.

It is obvious that there are twice as many unknown model parameters than in the previous step, i.e. every model parameter in each model is defined by Eq. 2, however, only one set of parameters is required to describe the swelling process over time. In this examination, the mean absolute difference was also needed to fit the functions to different time instants. In this case the extreme value problem solver determined the minimum for the average of the mean absolute difference:

$$
\bar{E}=\frac{\sum_{t=1}^{n_{t}} E_{t}}{n_{t}}
$$

where the average of the mean absolute difference is denoted by $\bar{E}$ and the number of time instants by $n_{t}$.

\section{Results and analysis}

The zero distribution was evaluated by MATLAB based on a picture taken from a sample of polymer beads. The sample consisted of approximately five hundred beads within the diameter range of 0.2 to $0.9 \mathrm{~mm}$. The probability distribution with regard to the size of the polymer beads is shown in Fig. 2.

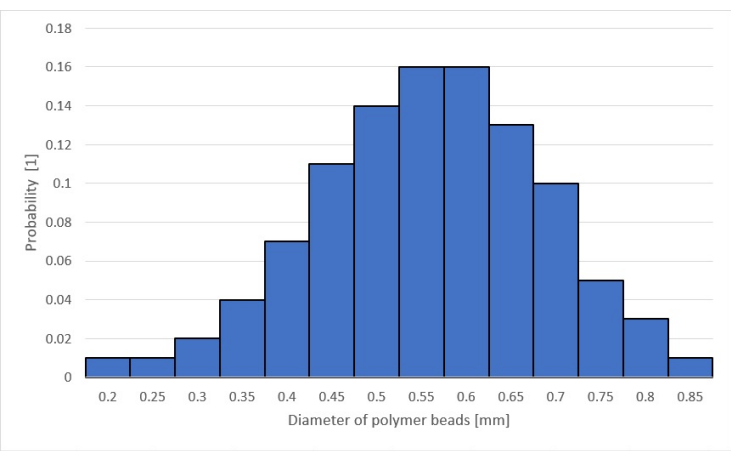

Figure 2: The probability distribution with regard to the size of the polymer beads before swelling within the diameter range of 0.2 to $0.9 \mathrm{~mm}$.

The parameters for the generated data were $p=2$ and $t=1500 \mathrm{~s}$ which means the polymer beads doubled their size in $1500 \mathrm{~s}$ and the steady-state size of the beads is this rate of growth. PSD was calculated twenty-five times between 0 and $1500 \mathrm{~s}$. Three of the twenty-five distributions are shown in Fig. 3.

All 23 models were fitted to all the distributions generated at every instant. Altogether twenty-three times twenty-five curve fittings were performed. The parameters were calculated for all the fitted models. For every fitted value a mean absolute difference $\left(E_{t}\right)$ was determined, the average of which are shown in Table 2

According to the values in Table 1 a selection of PSD models could be made. Those models show good agreement with the generated PSD, which yields an average mean absolute difference of less than five percent. A difference can be observed between the two methods to find extreme values. The global finder "NOMAD" has found a better fit for the two-parameter model known as BEST. In most cases the two search methods give the same results using the same model parameters. In some models, the global optimizer has found a better solution than was expected.

Eleven of the twenty-three models were found to be able to describe the distribution at all time instants. From

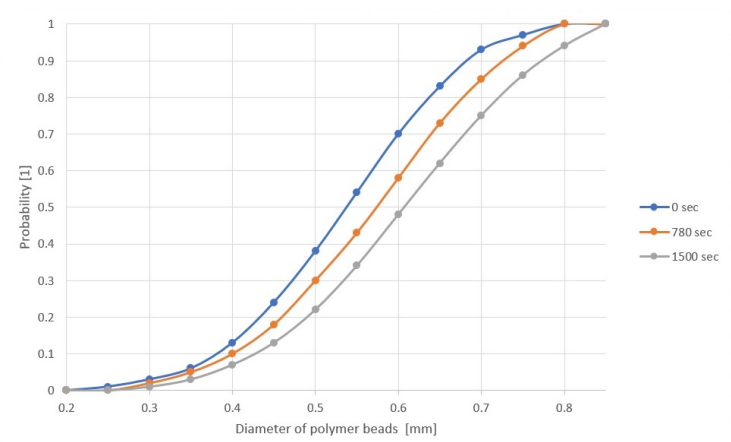

Figure 3: Cumulative distribution functions at three different time instants during the swelling of the polymer beads. 
Table 2: The goodness of fit for the different models.

\begin{tabular}{ccc}
\hline $\begin{array}{l}\text { PSD Model } \\
\text { ID }\end{array}$ & $\begin{array}{l}\text { SIMPLEX } \\
\text { fitting } \bar{E}[\%]\end{array}$ & $\begin{array}{l}\text { NOMAD } \\
\text { fitting } \bar{E}[\%]\end{array}$ \\
\hline 1 & 15 & 15 \\
2 & 39 & 39 \\
3 & 24 & 24 \\
4 & $\mathbf{2}$ & $\mathbf{2}$ \\
5 & 10 & 10 \\
\hline 6 & 12 & 8 \\
7 & $\mathbf{3}$ & 3 \\
8 & $\mathbf{1}$ & $\mathbf{1}$ \\
9 & 19 & 13 \\
10 & 8 & 9 \\
11 & 39 & $\mathbf{2}$ \\
12 & $\mathbf{5}$ & $\mathbf{5}$ \\
13 & $\mathbf{5}$ & $\mathbf{5}$ \\
14 & 12 & 12 \\
15 & 8 & 8 \\
16 & $\mathbf{1}$ & $\mathbf{1}$ \\
17 & 30 & 30 \\
\hline 18 & 35 & 13 \\
19 & $\mathbf{0}$ & $\mathbf{0}$ \\
\hline 20 & $\mathbf{2}$ & $\mathbf{1}$ \\
21 & $\mathbf{1}$ & $\mathbf{1}$ \\
22 & 37 & 34 \\
23 & $\mathbf{1}$ & $\mathbf{1}$ \\
\hline
\end{tabular}

these, the best model was the Weibull-3par which is shown in Fig. 4. This result does not mean that the model is able to describe polymer growth. A correlation should be present in terms of changing the parameters.

By examining the correlation of model parameters in these eleven models, one model called Jaky exhibited a correlation as shown in Fig. 5 The other models did not show any kind of correlation over time.

The Jaky model consists of one parameter and it seems to describe properly the growth of heterodisperse polymer systems. The other models did not show any correlation in terms of the changing of the parameter over time. In most cases one parameter was present which did

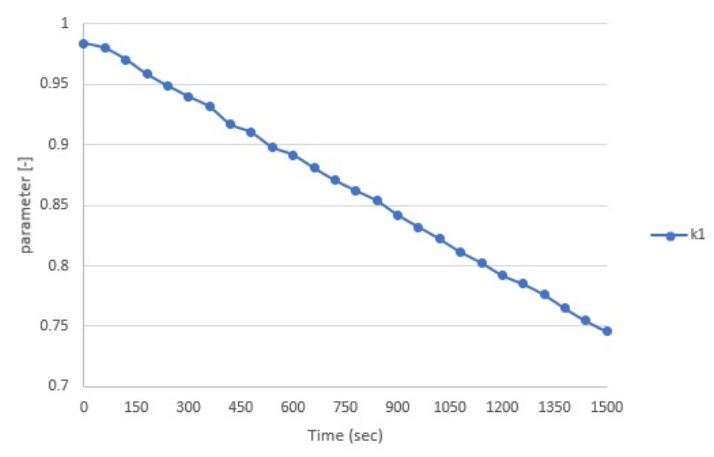

Figure 4: The change in the parameter of the Weibull-3par model after various durations of swelling.

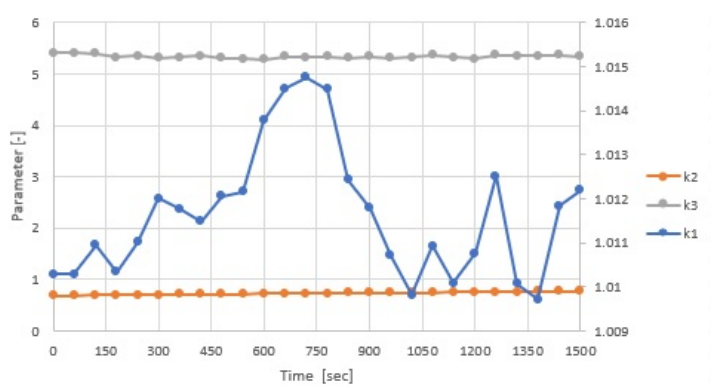

Figure 5: The change in the parameter of the Jaky model after various durations of swelling.

not show any regularity as is shown in Fig. 4.

The following examination sought to set a correlation between the change in the parameter and time due to the swelling of beads. With linear criteria (see Eq. 2) the goodness of fit will definitely deteriorate. The presumption was to find a model which exhibits a goodness of fit of under five percent after setting the criterion.

The results collected in Table 3 show that two of the eleven selected models produced a goodness of fit of under $5 \%$, the others were all in excess of $30 \%$. Therefore, the Jaky model consisting of one parameter and the Rosin-Rammler model of two exhibit a linear correlation for their parameters over time. The other nine models did not exhibit a linear correlation in their parameters over time. It would be worthwhile trying other non-linear functions to describe the parameters.

\section{Conclusion}

In polymer technologies, one of the steps is the swelling of the polymer beads. Several studies were conducted that deal with the swelling of polymer networks. Our aim was to examine the swelling of the polymer beads from a different point of view to find out if a simple PSD model exists which may be able to describe changes in size of this system.

Table 3: The goodness of fit of the linear correlation of parameters over time.

\begin{tabular}{ccc}
\hline $\begin{array}{l}\text { PSD Model } \\
\text { ID }\end{array}$ & $\begin{array}{l}\text { SIMPLEX } \\
\text { fitting } \bar{E}[\%]\end{array}$ & $\begin{array}{l}\text { NOMAD } \\
\text { fitting } \bar{E}[\%]\end{array}$ \\
\hline 4 & 2 & 2 \\
7 & 33 & 14 \\
8 & 1 & 1 \\
11 & - & 44 \\
12 & 44 & 44 \\
13 & 53 & 44 \\
16 & 44 & 44 \\
19 & 35 & 35 \\
20 & 35 & 35 \\
21 & 30 & 52 \\
23 & 44 & 44 \\
\hline
\end{tabular}


PSD models were examined according to their ability to describe the swelling of polymer beads. The results show that two models were present which could be used to describe and predict the behavior with regard to changes in size of the heterodisperse polymer bead systems.

In this examination, the copolymer styrene divinylbenzene was used. Two extreme value problem solvers were used to identify and confirm PSD models that are able to describe changes in size of the copolymer system.

Two models, namely the one-parameter Jaky model and the two-parameter Rosin-Rammler model, were able to describe the growth in size over time with an error of less than $2 \%$. The parameters of these models could be interpreted by a linear correlation over time according to the generated data that was produced in this study. Based on these working models a prediction can be made with regard to changes in size of a heterodisperse copolymer system over time.

\section{Symbols}

$\begin{array}{ll}E_{t} & \text { Mean absolute difference } \\ \bar{E} & \text { Average of mean absolute difference } \\ P(d)_{t}^{\prime} & \text { Calculated distribution } \\ P(d)_{t} & \text { Generated distribution } \\ n_{d} & \text { Number of data } \\ n_{t} & \text { Number of time instants } \\ k_{x} & \text { Parameters of models }\end{array}$

\section{Acknowledgment}

This research was supported by the Széchenyi 2020 project GINOP 2.2.1-15-2017-00059.

\section{REFERENCES}

[1] Painter, P.C.; Shenoy, S.L.: A simple model for the swelling of polymer networks, J. Chem. Phys., 1993 99(2), 1409-1418 DOI: 10.1063/1.465385

[2] Schott, H.: Swelling kinetics of polymers, J. Macromol. Sci., Part B: Physics, 1992 31(1), 1-9 DOI: 10.1080/00222349208215453

[3] Sweijen, T.; van Duijn, C.J.; Hassanizadeh, S.M.: A model for diffusion of water into a swelling particle with a free boundary: Application to a super absorbent polymer particle, Chem. Eng. Sci., 2017 172, 407-413 DOI: 10.1016/j.ces.2017.06.045

[4] Bayat, H.; Rastgou, M.; Nemes, A.; Mansourizadeh, M.; Zamani, P.: Mathematical models for soil particle-size distribution and their overall and fraction-wise fitting to measurements, Eur. J. Soil Sci., 2017 68(3), 345-364 DOI: 10.1111/ejss.12423

[5] Bayat, H.; Rastgo, M.; Zadeh, M.M.; Vereecken, H.: Particle size distribution models, their characteristics and fitting capability, J. Hydrology, 2015 529, 872-889 DOI: 10.1016/j.jhydrol.2015.08.067

[6] Blickle, T.; Lakatos, B.G.; Ulbert, Zs.; Mihálykó, Cs.: The hyperbolic tangent distribution and its applications-I. mathematical foundations, Hung. J. Ind. Chem., 1998 26, 89-96

[7] Blickle, T.; Lakatos, B.G.; Mihálykó, Cs.; Ulbert, Zs.: The hyperbolic tangent distribution family, Powder Technology, 1998 97, 100-108

[8] Le Digabel, S.: Algorithm 909: NOMAD: Nonlinear Optimization with the MADS Algorithm, ACM Trans. Math. Softw., 2011 37(4), 1-15 DOI: 10.1145/1916461.1916468 